\title{
EREBEA
}

Revista de Humanidades

y Ciencias Sociales

NúM. 1 (2011), pp. 177-202

ISSN: 0214-0691

\section{LA TEATRALITÀ IMPLiCITA NEI SERMONI DI BERNARdino dA SiENA: INDAGINE SULLE PROPOSIZIONI INTERROGATIVE}

\author{
Valentina Berardini \\ Universidad de Macerata
}

RESUMEN

El artículo presenta los resultados de una investigación aún en marcha sobre la relación entre la predicación y el teatro en la baja Edad Media. Basándose en las recientes formas de entender la predicación y la escena, la autora propone una metodología para desvelar los elementos teatrales implícitos que se están insertos en los sermones medievales. De forma particular se centra en los sermones de Bernardino de Siena y en su utilización de la interrogación como mecanismo para que el predicador realice una especie de diálogo con su auditorio. Aunque quizás no se esperase respuesta, hacer preguntas siempre tenía la utilidad de dar vida a los sermones llamando la atención de los oyentes e implicándolos en el discurso.

Palabras Clave

Predicación, Teatro, Performance, Sermones medievales

Fecha de recepción: 2 de febrero de 2011 Fecha de aceptación: 1 de marzo de 2011

\section{Abstract}

The article presents the results of an ongoing research about the relationship between preaching and theatre in the late Middle Ages. Based on the recent developments of understanding preaching and performance, it proposes a methodology in order to uncover embedded theatrical elements which are implied in medieval sermons. In particular, it focuses on Bernardino da Siena's sermons and his use of questions as elements through which the preacher establishes a sort of dialogue with his audience. Perhaps no answer was expected but asking a question was useful in order to liven up the sermon by calling on the listeners' attention and involve them in the discourse.

\section{KeYwORDS}

Preaching, Theatre, Performance, Medieval sermons 



\section{Premessa}

Nella società medievale, caratterizzata da un elevato grado di oralità e da una scarsa circolazione libraria, la predicazione fu il più importante mezzo di comunicazione e diffusione delle conoscenze; infatti, attraversando l'Europa per divulgare il messaggio evangelico, condannando vizi ed elogiando virtù, i predicatori contribuirono alla circolazione delle notizie e delle idee. Tuttavia i sermoni non furono solo un momento importante della vita religiosa dei fedeli ma divennero spesso grandi eventi sociali di intrattenimento pubblico, come testimoniato dal successo che accompagnò figure quali Vicent Ferrer e Bernardino da Siena.

Il presente contributo intende mostrare alcuni risultati derivanti da una ricerca dedicata allo studio del rapporto fra predicazione e teatro ${ }^{1}$, due fenomeni che, nonostante la loro apparente opposizione, mostrano significativi punti di contatto. Tale connessione risulta particolarmente evidente nei sermoni di Bernardino da Siena $(1380$ - 1444) che, erede del modello omiletico proposto da Francesco d'Assisi, operò un sensibile rinnovamento delle forme dell'eloquenza sacra; in particolare, egli riuscì a far convergere nella predicazione le indicazioni delle artes praedicandi, quelle proprie dell'ars concionandi e i caratteri tipici delle mimesi teatrali, dando vita a delle vere e proprie rappresentazioni drammatiche basate sul continuo dialogo con il pubblico. D'altra parte, come ha notato Raoul Manselli, proprio il carattere dialogico costituiva la cifra caratteristica dello stile omiletico di Francesco: «la predicazione francescana (...) tendeva a evitare il sermone impostato sic et simpliciter, e ciò anche quando vi furono frati ordinati sacerdoti, ma ebbe sempre la tendenza ad entrare in dialogo con i fedeli, a presentarsi in stretto collegamento con loro, fino alla battuta di spirito, alla facezia pungente ma anche cordiale e affettuosa» ${ }^{2}$.

1 La ricerca cui si fa riferimento costituisce l'oggetto della mia tesi di dottorato, discussa nella primavera 2011 e intitolata La teatralità implicita. Aspetti performativi della predicazione di Bernardino da Siena (tutor: C. Delcorno, Alma Mater Studiorum, Università di Bologna). Sono grata ai lettori di Erebea che volessero darmi indicazioni e suggerimenti relativamente a elementi performativi nei sermoni medievali (valentina.berardini@gmail.com).

2 R. Manselli, Il francescanesimo come momento di predicazione e di espressione drammatica in $I l$ francescanesimo e il teatro medievale, Atti del Convegno Nazionale di Studi (San Miniato, 8-9-10 ottobre 1982), Castelfiorentino 1984, pp. 121 - 133, pp.128 - 129. 
I sermoni tramandati ci sono giunti come testi scritti e dunque privi della loro importante e specifica componente orale; è però possibile tentare di ricostruire tale componente concentrandosi sul testo e ricercando in esso dei segnali di un'esecuzione non solo orale ma anche e soprattutto teatrale.

L'indagine cui si è accennato è volta all'isolamento della componente drammatica dei sermoni al fine di valutare il grado di potenziale teatrale dei testi; con tale espressione si intende rilevare la maggiore o minore presenza di fenomeni che attribuiscono al testo una connotazione tale da lasciar supporre una originaria resa drammatica, se non integrale almeno limitata alle parti che contengono alcuni specifici elementi. Proprio la necessità di ricostruire la componente orale e teatrale, perduta a causa della trasmissione in forma scritta dei testi, induce a definire la teatralità dei sermoni come 'implicita': tale carattere non è esplicitamente dichiarato e tuttavia l'autore dei testi ricorre, più o meno consapevolmente, a strumenti derivati dall'ambito teatrale e capaci di rendere il discorso particolarmente attraente. In tale contesto, i fenomeni mutuati dall'ambito teatrale sono stati definiti 'indicatori' in quanto spie della componente drammatica del sermone. Gli indicatori di teatralità implicita possono essere definiti come elementi in grado di interrompere il tono didattico e teorico del sermone attraverso il ricorso a immagini e formule comunicative tipiche di altre espressioni verbali; la loro presenza suggerisce la sovrapposizione del codice comunicativo visuale e dialogico, tipico del teatro, al codice comunicativo verbale, caratteristico di ogni comunicazione orale. Ogni volta che il predicatore, per la divulgazione del messaggio evangelico, si serve di strumenti in grado di favorire il passaggio da un discorso astrattamente teorico e dottrinale a uno concreto, visivo, tangibile, che si avvicina alla messa in scena e alla mimesi, si può parlare di potenziale teatrale del testo. La selezione degli indicatori di teatralità implicita è quindi avvenuta in base alla loro capacità di alleggerire il peso teorico del sermone e di saperne tradurre visivamente il contenuto; la traduzione delle parole in immagini, oltre a favorire una migliore comprensione da parte degli ascoltatori, stimolava l'emotività, consentendo ai predicatori di raggiungere lo scopo di una persuasione non solo razionale ma anche e soprattutto emotiva.

Le riflessioni sulla la teatralità implicita nei sermoni bernardiniani non sono certo nuove e numerosi studiosi hanno già affrontato questo tema in anni passati, sottolineando la matrice fortemente teatrale dello stile oratorio di Bernardino da Siena; pertanto, la novità dell'analisi proposta non consiste tanto nel rilevare la presenza di una componente teatrale nelle prediche di Bernardino da Siena quanto piuttosto nel declinare tale componente, individuando con esattezza non solo i fenomeni da cui è costituita ma anche e soprattutto le caratteristiche di ciascun fenomeno. 


\section{Le Proposizioni INTERrogative NEI SERMONI di BERnARdino dA SienA ${ }^{3}$}

Le proposizioni interrogative costituiscono una classe di indicatori in quanto contribuiscono alla costruzione di un registro dialogico che coinvolge predicatore e pubblico; in particolare, Bernardino si serve di tale tipo di proposizioni per dare vita a dialoghi fittizi con i suoi ascoltatori in modo da rendere più vivace l'esposizione del sermone. Anche qualora le domande non siano accompagnate da una risposta riferibile all'interlocutore cui il predicatore si rivolge, è indubbio che le proposizioni interrogative testimoniano dell'esistenza di un registro dialogico. Le domande si configurano, infatti, quali elementi in grado di interrompere il monologo del predicatore, coinvolgendo direttamente gli ascoltatori. Molte domande prendono avvio da una richiesta esplicita rivolta dal predicatore a un interlocutore del pubblico affinché questi si pronunci, esprimendo la propria opinione sull'argomento discusso nel sermone. Se ne ha un esempio nella predica recitata il 27 marzo 1424:

Or dimmi una cosa. Qual credi tu che sia maggiore peccato, o d'una donna che si facci bella e adorna solamente per piacere agli uomini, sanza altro male pensiero, o andare a rubare e romei alla strada, o prestare a usura, o rubare vedove o pupilli? Rispondi che molto è maggiore peccato quello della donna che non è el rubare perché la fa maggior danno. ${ }^{4}$

Sebbene Bernardino costruisca l'intera proposizione con lo scopo di indirizzare la risposta del pubblico a suo favore e sebbene, di fatto, non conceda la parola ai suoi ascoltatori, è importante rilevare il tentativo di coinvolgimento dei fedeli attraverso l'invito perentorio a rispondere alla sua domanda. Infatti, la proposizione che apre il periodo è contraddistinta dalla presenza di un verbo imperativo (dimmi) che costringe gli interlocutori a porre particolare attenzione a quanto il predicatore sta per dire, impegnandoli a ragionare per poter soddisfare la richiesta di Bernardino.

Le interrogative costituiscono un elemento costante e centrale nella tecnica oratoria bernardiniana che vi ricorre con altissima frequenza arrivando alla costruzione di sezioni di testo scandite interamente dal susseguirsi di domande rivolte al pubblico, come dimostra il seguente passo:

3 Per snellire i riferimenti bibliografici, le citazioni dei sermoni bernardiniani si avvalgono delle seguenti sigle: Bernardino da Siena, Le prediche volgari, edite da C. Cannarozzi, Tip. Pacinotti, Firenze, 1934 (F24); Bernardino da Siena, Le prediche volgari edite da C. Cannarozzi, Tip. Pacinotti, Pistoia, 1940 (F25); Bernardino da Siena, Prediche volgari sul Campo di Siena, 1427 a cura di C. Delcorno, Rusconi, Milano, 1989 (S27). Alla sigla segue l'indicazione, in cifre romane, del numero del sermone, del volume di riferimento e delle pagine corrispondenti alla citazione.

4 F24, XXL, vol. I, p. 334. 
Come ti fiderai tu d'una persona che tu non conosca? Come crederai tu cosa che tu non l'oda? Cosi come puoi tu conoscere Iddio se tu non lo conosci? Come lo puoi ubbidire, se tu non sai e suoi comandamenti e quello che di lui debbi credere? Bisognati sapere e dodici articoli della fede che si contengono nel Credo piccolo o grande e così ài 'l fondamento della fede iscoperto'.

In un altro sermone, recitato nel corso dello stesso ciclo, Bernardino ricorre a un procedimento simile. Infatti, discorrendo della giustizia dei peccati davanti a Dio, egli costruisce una serie incalzante di proposizioni interrogative accompagnate, ciascuna, dalla sua personale risposta:

Credete voi ch'e peccati abbino mutato linguaggio e usanza? Sempre gridano a Dio vendetta, e quanti maggiori sono e più scellerati, tanto più gridano di forza. Che grida credete che sia dinanzi da Dio? Del maledetto vizio de' sottomiti contro a natura. Che credete? De' bestemmiatori. Che grida credi vi sia? Dell'usure tolte e delle vedove e pupilli rubati. Che grida vi sono? De' vostri sottoposti male trattati e rubati. Ogni peccato grida vendetta. ${ }^{6}$

Analogamente, nella predica recitata a Siena il 23 agosto, prima di congedare il suo pubblico, il santo formula una serie di domande volte a fissare l'attenzione degli ascoltatori sul momento della comunione, troppe volte considerato con eccessiva leggerezza:

Terza è comunione. Quando tu ti va a comunicare, non consideri tu che vai a pigliare e ricevare il vero corpo di Cristo? O iniquo uomo, con che bocca vi vai? Con che cuore vi vai? Con che occhi vi vai? Con quali mani vi vai? Con quali piei tu vi vai?

Come si vede, in tre righe di testo, Bernardino ha inserito sei proposizioni interrogative che non lasciano nemmeno il tempo della risposta e mettono in luce l'eccessiva approssimazione dei fedeli nel compiere un atto importante quale è quello di ricevere il sacramento della comunione. Il ritmo è reso inoltre ancor più incalzante dal ricorrere dell'epifora "vi vai» che conclude tutte le interrogative, ad eccezione della prima. La ripetizione, quasi ossessiva, della formula interrogativa serve a mettere in risalto la moltitudine dei peccati commessi dall'uomo destinati a ricevere la punizione divina. Analogamente a quanto era avvenuto nell'esempio precedente le domande si susseguono in modo rapido e veloce soffocando ogni tentativo di sottrarsi all'evidenza dimostrata dal predicatore. 
A livello linguistico, le domande si configurano come atti illocutori in quanto il parlante, nel formularle, compie un'azione ben precisa: chiedere un'informazione. Tale richiesta può essere animata da scopi diversi la cui individuazione esplicita il senso e la funzione del ricorso a una proposizione di tipo interrogativo. D'altra parte, il ricorso a formule interrogative è perfettamente in linea con l'urgenza espressiva tipica della tecnica omiletica bernardiniana, impegnata nella costante creazione di un rapporto diretto con il pubblico attraverso il quale coinvolgere attivamente gli ascoltatori. La consapevolezza di trovarsi di fronte a un artificio messo in atto per interrompere il monologo del predicatore offre una chiave di lettura importante per poter ricondurre l'al to numero di proposizioni interrogative a una serie di classi, funzionali a metterne in luce caratteristiche e scopi. Prendendo avvio dalle riflessioni svolte da Piero Sollazzi ${ }^{8}$, l'analisi è stata condotta adottando come principio distintivo la funzione svolta dalle interrogative all'interno del progetto comunicativo del predicatore. Si distinguono quindi sette classi: 1) proposizioni interrogativo-assertive accompagnate dalla risposta di Bernardino; 2) proposizioni interrogative con valore esclamativo; 3) proposizioni interrogative con implicita risposta del pubblico; 4) proposizioni interrogative esplicative; 5) proposizioni interrogative retoriche; 6) proposizioni interrogative con funzione fatica; 7) proposizioni interrogative correttive. Inoltre all'interno della prima classe è possibile individuare un sottogruppo costituito dalle proposizioni interrogative con valore di esortazione o di consiglio.

\section{Proposizioni interrogativo-ASsertive acCompagnate} DAlla Risposta di BERnardino

L'accumularsi di proposizioni interrogative potrebbe apparire poco consono alla natura di un testo concepito con lo scopo di "convincere, esortare» ${ }^{9}$ e nel quale ci si aspetterebbe di trovare «una preponderanza di frasi assertive, imperative, esclamative» ${ }^{10}$; in realtà tali enunciati non mancano ma assumono spesso la forma di «interrogative interne, di quel tipo cioè particolare di interrogazioni che, tali da un punto di vista formale, non lo sono da un punto di vista del senso» ${ }^{11}$. Si prenda ad esempio la seguente proposizione: "Che ànno bisogno gli uomini che vogliono incominciare a fare bene? Di quattro cose» ${ }^{12}$; dietro l'aspetto interrogativo, si cela, in realtà, un enunciato dichiarativo del tipo: «Gli uomini che vogliono incominciare a fare bene hanno bisogno di quattro cose». Dal confronto risulta evidente come a parità di valore semantico, la prima formulazione risulti molto più espressiva

8 P. Sollazzi, Meccanismi allocutivi in S. Bernardino in "Miscellanea Francescana», t.80, fasc. III-IV (Luglio-Dicembre 1980), pp. 385 - 424.

9 Ibidem, p. 416.

10 Ibidem

11 Ibidem

12 F24, XL, vol. II, p. 194. 
della seconda e dunque più efficace dal punto di vista comunicativo. Scopo di tali domande è quindi quello di conferire dinamicità e movimento al discorso; ciò appare tanto più vero se si considera che si tratta, per lo più, di interrogative cui si accompagna una risposta esplicita dello stesso predicatore. In pratica, obiettivo della domanda non è ottenere una risposta dal pubblico ma semplicemente rivestire di una forma più espressiva un enunciato assertivo. Ad esempio, nella predica recitata il 10 marzo 1424, mentre discute dei pericoli connessi al commettere peccato, Bernardino si rivolge ai suoi ascoltatori e chiede: «Sai perché non sà in odio il peccato? Perché non si intende la colpa. E perché non si intende la colpa? Perché non si ripensa, e però dice recogitabo» ${ }^{13}$. Il predicatore poteva certamente sostituire le due proposizioni interrogative con enunciati dichiarativi dal medesimo valore semantico; la scelta operata va, però, nella direzione di una maggiore espressività, capace di richiamare l'attenzione del pubblico. Sebbene sia lo stesso Bernardino a rispondere, gli ascoltatori sono impegnati, anche solo per una frazione di secondo, nel trovare una risposta adeguata alla domanda del predicatore.

Il coinvolgimento del pubblico risulta ancora più evidente nei casi in cui Bernardino costringe i suoi ascoltatori a riflettere sul senso della sua predicazione, adottando una visione di insieme dell' intero ciclo e collegando fra loro i temi affrontati nei diversi sermoni. Ad esempio, in apertura della predica recitata il 19 marzo 1424, il santo esplicita il legame esistente fra quanto predicato il giorno precedente e il tema che è in procinto di trattare: "Perché nel dì d'ieri io narrai la storia del santo evangelio? Per serbarvi oggi materia molto utile» ${ }^{14}$. In questo modo, ogni esposizione acquista senso non solo come trattazione singola ma anche in riferimento all'intero programma omiletico di cui Bernardino rende partecipi i suoi uditori.

La predilezione per proposizioni interrogative capaci di esprimere in maniera più espressiva un contenuto di tipo dichiarativo ritorna anche nel ciclo del 1425 dove, in più occasioni, Bernardino formula una serie di domande cui egli stesso fornisce una risposta. È quanto avviene nel sermone recitato il 9 febbraio dove, discorrendo delle necessità temporali che guidano l'agire umano, il predicatore domanda:

Quando ài desiderio di mangiare o bere, chi tispira a quello cotale desiderio e voluntà?Non è Iddio, non l'angelo, non la tua virtù, non il dimonio, non la tua nequizia. Chi lo fa? La nicissità della naturale vita così ordinata ${ }^{15}$.

Ancora, nella predica recitata il giorno seguente, il santo, sfruttando la formula interrogativa, offre una definizione dell'atto della preghiera e del significato che questa assume: "Che cosa è orare? Ė a Dio la mente levare» ${ }^{16}$. 
Il sermone sulla comunione recitato il 5 aprile 1425 offre nuovamente lo spunto per la formulazione di domande volte a mettere in rilievo la disposizione spirituale che deve accompagnare il sacramento in questione: "Con che si piglia il Corpo di Cristo? Colla bocca? No, ma pigliasi con cuore, coll'anima di drento» ${ }^{17}$. Un procedimento analogo si ritrova anche nel sermone recitato nella Pasqua del 1425 dove viene messo in rilievo il valore della Resurrezione quale necessario complemento al sacrificio di Cristo: "Che vale a noi il suo incarnare meraviglioso, che vale a noi la sua dura e aspra passione e morte se non ci fussi suta la resurrezione? Nulla! [...] Quale fu la possanza di Cristo? La sua morte» ${ }^{18}$.

Il ciclo senese non fa eccezione anche se, come si vedrà, è più ricco di interrogative di altro tipo, funzionali al raggiungimento di scopi diversi rispetto alla pura valenza informativa che caratterizza la prima classe di domande oggetto di analisi. Nel sermone recitato l'8 settembre, trattando dell'incarnazione del Cristo, Bernardino ricorre a una proposizione interrogativa con la quale sottolinea il legame tra il Salvatore e la Vergine Maria:

El corpo di Cristo che è sopra a tutti i sacramenti de la Chiesa, donde è venuto? Venne dal ventre della gloriosa Vergine Maria; e forse che voi non vi pensaste mai più che il corpo di Cristo consacrato è proprio de la carne de la Vergine Maria ${ }^{19}$.

E così ancora, nel sermone dedicato ai peccati capitali, individuata nella scomunica una delle conseguenze dell'avarizia, il predicatore chiede:

La scomunicazione che credi tu che ella sia? Sappi che la scomunicazione è il maggior fatto che si possa fare in questo mondo. Ella ti fa serrare la porta di vita etterna, che non vi può entrare niuno scomunicato ${ }^{20}$.

1a. Proposizioni interrogative CON VALORE di esortaZione o di CONSIGLio

Tra le proposizioni interrogative accompagnate dalla risposta esplicita offerta dallo stesso predicatore possono essere incluse anche quelle domande formulate con lo scopo di consigliare gli ascoltatori riguardo il migliore comportamento da adottare o di esortare il pubblico a compiere determinate azioni; la risposta del predicatore offre quindi l'esortazione o il consiglio annunciati nella formulazione della domanda.

Così ad esempio, nel sermone recitato il 21 marzo 1424, nell'invitare i suoi fedeli ad astenersi dal compiere azioni a danno di quanti sono reputati cattivi,

17 F25, LXI, vol. III, p. 339.

18 F25, LXIV, vol. III, pp. 400 - 401.

19 S27, XXIV, vol. I, p. 704.

20 S27, XXXV, vol. II, p. 1030. 
Bernardino formula una domanda che contiene già l'annuncio di un consiglio che egli sta per dispensare al suo pubblico: "Vuo' tu fare grande ingiuria a' cattivi sanza peccato, ma con merito? Or fa' di dare quanto favore tu puoi a' buoni $\rangle^{21}$. Nel sermone recitato due giorni dopo, il 23 marzo, il predicatore ricorre nuovamente a una domanda per esortare i suoi fedeli a considerare con attenzione il modo di vestire degli uomini al fine di intuirne i vizi: «Volete voi conoscere ne popoli quello e quell'altro? Guardateli ne' vestimenti loro. Chi veste pomposo è sempre vizioso» ${ }^{22}$. Analogamente, nel sermone del 24 aprile 1424 si legge: "Vuoi tu conoscere el Creatore? Or fa' di conoscere le creature e, d'esso fatto, per questo conoscerai el Creatoren ${ }^{23}$.

Interrogative di questo tipo sono più rare nel ciclo del $1425 \mathrm{ma}$ è possibile individuarne un esempio nel sermone recitato il 4 aprile; nel presentare le caratteristiche dello stato spirituale, Bernardino offre un consiglio a quanti, fra i suoi fedeli, desiderino raggiungere lo stato di perfezione: "Or vuoi tu doventare

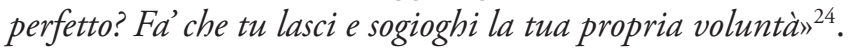

L'affinarsi dell'arte omiletica bernardiniana è testimoniata, nel ciclo senese, dal più frequente ricorso alle proposizioni interrogative. Tra queste è possibile identificare anche un buon numero di domande nate con lo scopo di consigliare o esortare il pubblico, come risulta evidente dal seguente esempio: "Vuoi vedere quanta è questa sua misericordia? Va' legge Matteo al XVIII capitolo, dove disse a santo Pietro: "Perdona al peccatore, non septies, sed septuagies septies" ${ }^{25}$. La citazione è funzionale a rimarcare la grandezza della misericordia divina per confermare la quale Bernardino ricorre a un'auctoritas. Anche in un'altra occasione il santo rinvia i propri fedeli alla lettura di un testo autorevole, i Dialogi di Gregorio Magno, per trovare conferma di quanto andava affermando:

Vuoi vedere quanto dispiace a Dio usare il matrimonio per libidine? Legge nel Dialago, dove tu trovarai che essendo ordinata una processione, e uno avendo usato il matrimonio in atto di carnalità, facendosi la processione, gli entrò uno diavolo adosso. Ha'la intesa?. ${ }^{26}$.

Il riferimento al testo di Gregorio è incastonato tra due proposizioni interrogative delle quali l'una esorta a verificare la veridicità delle parole del predicatore, l'altra vuole accertarsi dell'effettiva comprensione della teoria espressa. Più orientata a fornire un consiglio di tipo pratico che serva da guida nella condotta di vita 
quotidiana è, invece, l'interrogativa formulata nel corso del sermone recitato il 15 settembre: "Vuoi tu fare a mio modo, o fanciulla? Or fa' che tu ti guardi da madonna Dimestica» ${ }^{27}$. In questo caso, Bernardino, discorrendo delle virtù della Vergine, si rivolge direttamente alle fanciulle presenti fra il pubblico, esortandole ad assumere un atteggiamento riservato. La stessa esortazione è ribadita, poco dopo, facendo ricorso, ancora una volta, a una proposizione interrogativa:

A queste tali cose debbi tenere l'urecchie uperte: non cosi quando tu odi o canti o suoni de' giovani che ti vogliono far mal capitare. Quando tu li senti, sai che fa'? Fugge, non gli stare a udire, però che se tu vi starai, tu starai a gran pericolo, ché l'animo de la fanciulla vagilla, non sta saldo, e poi si viene..et cetera ${ }^{28}$.

\section{Proposizioni interrogative con valore esclamativo}

Sebbene ricorrano con frequenza minore rispetto ad altre, vi sono alcune proposizioni interrogative che, oltre ad avere un colorito retorico, assumono anche un valore esclamativo. In esse il predicatore si mostra stupito di fronte all'evidenza di certi comportamenti e traduce tale incredulità in una domanda capace di coinvolgere anche i suoi ascoltatori, posti di fronte all'assurdità del fatto in questione. Se ne riportano, di seguito, alcuni esempi:

Doh, che pazzia è questa di coloro che vogliono sapere più che Dio non vuole che si sappia? Chi è colui che il sa? (S27, III, vol. I, p. 152)

Dob! Che cosa è ella, che talvolta una fanciulla savia, acostumata, buona, bella, con molte virtù, s'abbattarà a uno che non ha virtù niuna, che meritarebbe d'essar moglie d'uno imperatore? (S27, XIX, vol. I, p. 558)

Oimè che stemperanza è questa? ( S27, XX, vol. I, p. 589)

È importante rilevare, in tali esempi, il ricorrere della particella «Doh!», indicativa di una proposizione esclamativa accompagnata, forse, da un gesto capace di tradurre in riferimento concreto l'esclamazione realizzata verbalmente.

\section{Proposizioni interrogative con implicita risposta del pubblico}

Si è già avuto modo di evidenziare il ricorso alla mimesi di espressioni del pubblico, funzionale a creare dialoghi in grado di coinvolgere attivamente gli ascoltatori. In tale contesto, assumono particolare importanza quelle proposizioni interrogative accompagnate da una risposta breve e sintetica, generalmente costituita dalla particella affermativa 'sì': 
Doh, avete voi mai veduto pescare a lenza? - Si. - Elli si piglia uno lombrico, e mettesi nell'amo, e 'l pesce va per pigliare el lombrico, e rimane preso lui. Vedi che per avere il cibo rimane preso il pesce $e^{29}$.

La risposta all'interrogativa pone alcuni problemi di interpretazione dal momento che può essere letta sia come mimesi dell'espressione del pubblico sia come anticipazione, da parte di Bernardino, del consenso del pubblico sia, infine, come reazione del predicatore a una domanda di tipo retorico. Nello specifico dell'esempio citato, infatti, appare piuttosto ovvio che le persone cui Bernardino si rivolge siano a conoscenza delle modalità proprie della pesca. Secondo Piero Sollazzi, casi di questo genere rappresentano un'ulteriore attestazione dell'importanza rivestita dall' «elemento-pubblico» ${ }^{30}$ con cui Bernardino costruisce costantemente un dialogo, più o meno ideale: "poiché questa "sermocinatio" non può, per forza di cose, essere un colloquio, ecco che l'ipotetica risposta del pubblico è spesso surrogata da un "sì", semanticamente neutro, che costituisce in sostanza una semplice appendice della domanda ${ }^{31}$. Se si accetta l'ipotesi che si tratta di una riproduzione, seppure surrogata, dell'espressione del pubblico, è però doveroso rilevare che Bernardino ricorre a una mimesi fortemente depotenziata, limitata alla pronuncia di una particella affermativa. La forza mimetica, tipica delle riproduzioni analizzate nel paragrafo precedente, risulta a tal punto attenuata da far dubitare, a mio avviso, che si tratti di una vera e propria imitazione di un'espressione altrui. D'altra parte, l'esempio in questione non è del tutto estraneo a una coloritura retorica in forza della quale la particella affermativa si configura quale unica e logica risposta alla domanda formulata. Anche qualora venga meno il valore retorico, assente del resto in molte altre interrogative di questo tipo, rimane tuttavia possibile interpretare l'affermazione come anticipazione della risposta che l'interlocutore è in procinto di pronunciare; ecco allora che il "si" più che riproduzione fedele, mimesi, dell'espressione del pubblico viene ad assumere l'aspetto di interpretazione della reazione degli ascoltatori. È come se Bernardino, consapevole del pensiero del pubblico, formulasse una domanda di cui egli stesso conosce già l'eventuale risposta da parte dei suoi fedeli; per questo non aspetta che siano loro a rispondere ma interviene lui stesso, conscio di allinearsi perfettamente alla posizione dei suoi ascoltatori.

Prima di proseguire nell'analisi delle interrogative di questo tipo, è necessario prenderne in esame alcuni esempi:

a) O tu che non udisti mai predica, secci venuto? - Sì. - O pone mente a questi quatro splendori acciò che tu sia alluminato e dichiarato per li tempi a veni-

29 S27, III, vol. I, p. 164.

30 P. Sollazzi, Meccanismi allocutivi in San Bernardino...cit., pp. 418 - 419

31 Ibidem 
re, e perché ti venga voglia d'andare a udire la predica e la parola di Dio da ora inanzi. (S27, III, vol. I, p. 147)

b) Vuoi maritare bene la tua figliuola? E tu, uomo, vuoi pigliare donna? - Si Or fa' che queste tre cose siano nella donna [...] (S27, XIX, vol. I, p. 541)

c) Hami tu anco inteso? - Si - io non vi parlo in francioso. (S27, IV, vol. I, p. 200)

d) O giovano, è mormorato di te? - Sì. - Cagione ci è. E tu, donna, è mormorato di te? - Si. (S27, VIII, vol. I, p. 288)

e) Vuoi ch'io te lo insegni? - Si. (S27, XX, vol. I, p. 598)

f) Doh, vuoi fare a mio modo? In nel nome di Dio, ha detto male di te? - Si! - Or non volere cercare: lassalo andare; tura li occhi, tura l'orecchie e ogni sentimento, e non cercare più che questo, che tu nol sai: dico che viene da Dio. (S27, VIII, vol. I, p. 299)

g) Donna, vuoi piacere a tuo marito? - Si. - Or parla poco; [...] (S27, IX, vol. I, p. 307)

h) Hai tu detto male d'uno? - Sì. (S27, IX, vol. I, p. 322)

i) Dici il patarnostro? - Si. (S27, IX, vol. I, p. 322)

1) O perché lo fece tanto grande il giudicio suo? Nol sai? - No. [...] (S27, XIII, vol. I, p. 408)

m) Hai tu veduti questi sei pericoli e peccati? - Si. - Che ci resta? Sai che? Aspetta il flagello di Dio, e vede l'ultimo. (S27, XIII, vol. I, p. 410)

n) Vuo'lo vedere? Ma dimi, l'uomo non fu elli creato da Dio di fango? - Si. (S27, XIX, vol. I, p. 560)

o) Se Idio venisse a mangiare con teco, farestili tu onore? - Sì. (S27, XXII, vol. I, p. 643)

Considerata la varietà degli esempi, appare piuttosto difficile giungere a conclusioni generali, capaci di comprendere al proprio interno tutte le possibili occorrenze di interrogative accompagnate da una risposta costituita da una semplice particella affermativa. Se da una parte è possibile rilevare la presenza di proposizioni interrogative cui segue, quasi certamente, la risposta del pubblico (a, b, d, e, f, g, h, i, o $)^{32}$; dall'altra, è ravvisabile il ricorso a domande dal colorito retorico capaci di orientare la risposta del pubblico e di predisporre quindi l'andamento del discorso (c, $1, \mathrm{~m}, \mathrm{n}$ ). In quest'ultimo caso, in assenza di altri elementi, è difficile capire se Bernardino risponde in veste di uditore o in veste di predicatore; del resto, non è escluso che la risposta non sia altro che una semplice, logica e ovvia appendice della domanda.

Come ha giustamente sottolineato Piero Sollazzi, a volte, la particella affermativa non costituisce una vera e propria risposta, quanto piuttosto un «trait-d'union tra i membri componenti una costruzione paratattica, la quale

32 Si tratta ovviamente della risposta riprodotta dal predicatore che, quindi, mette in atto un meccanismo proprio della sermocinatio. 
corrisponde, da un punto di vista logico, ad una struttura ipotattica» ${ }^{33}$. A volte, infatti, gli enunciati nascondono dei periodi ipotetici, come attestato dal seguente esempio: "Vuo'la fare gialla l'infamia? - Si - Or sputavi su con buona carità» ${ }^{34}$. A volte, afferma ancora Piero Sollazzi, «il significato ipotetico trapassa in quello assertivo, con in più un forte senso esortativo» ${ }^{35}$, come avviene nella predica recitata il 17 agosto: "O cittadini, volete campare Siena? - Si - fa' che tu oda la parola di Dio; fate mandare il bando, che insino che sia detta la predica ogni mattina, niuno apra la buttiga» ${ }^{36}$.

\section{Proposizioni interrogative esplicative}

Un'altra categoria di proposizioni interrogative è rappresentata da tutte quelle domande formulate per spiegare e precisare il senso di una citazione, di una metafora o di un'allegoria inserita dal predicatore all'interno del proprio discorso. Al fine di sollecitare l'attenzione degli ascoltatori, impegnandoli in uno sforzo ermeneutico, Bernardino formula delle domande che spezzano la monotonia della spiegazione dotta e consentono, al tempo stesso, di proseguire l'operazione didattica avviata; infatti le interrogative, pur vivacizzando il tono del sermone, non concedono alcuna vacanza alla mente dell'ascoltatore, piuttosto lo impegnano, in prima persona, nella decodificazione del significato della citazione, della metafora o dell'allegoria. Si prenda ad esempio il sermone recitato il 7 aprile 1424 a Firenze che si apre con la consueta citazione dal Vangelo:

Domine iam fetet quatriduanus est. Scribitur Iovanne duodecimo capitulo in nel vangelio odierno. In volgare disse Marta a Gesù: "Signore, e’ pute, egli è stato già quattro di morto, nel monimento seppellito. E' pute; non lo iscoprire! E seguita stamane la sesta illuminazione di fetore. ${ }^{37}$

Concluso l'annuncio relativo al tema dell'esposizione, Bernardino si rivolge direttamente ai suoi ascoltatori e chiede:

Di cui parla Marta a Gesù? Di Lazzero, già quattro dì nel sepolcro seppellito. Chi è Lazzero? È 'l popolo di Firenze. Io predico a voi e none a' tedeschi, tutta la congregazione de' soddomiti, Lazzero puzzolente a Dio e al mondo. In quattro modi si pecca. ${ }^{38}$

33 P. Sollazzi, Meccanismi allocutivi in S. Bernardino...cit., p. 419.

34 S27, VIII, vol. I, p. 297.

35 P. Sollazzi, Meccanismi allocutivi in S. Bernardino...cit., p. 419.

36 S27, III, vol. I, p. 160.

37 F24, XXXI, vol. II, p. 37.

38 F24, XXXI, vol. II, p. 37. 
Le domande invitano gli ascoltatori ad assumere un ruolo attivo, partecipando al discorso del predicatore e ponendosi degli interrogativi relativi a quanto viene loro detto; nello stesso tempo, le due proposizioni interrogative riescono nell'intento di modificare il ritmo del sermone, spezzandone la monotonia e ravvivandone l'andamento. Poco più avanti ricorre un esempio analogo, applicato, stavolta, a un'allegoria:

Dicono alcuni dottori che le ape non fanno mai il mele ove fusse in uno vallone il rimbombo dell'acque o delle boci. Che vuole dire il rimbombo dell'acque e delle boci? Non altro se no la cattiva usanza e le cattive e disoneste parole che ti rimbombano negli urecchi. Non vi si può fare mele di dolcezza. ${ }^{39}$

Nel ciclo del 1425 ricorrono procedimenti analoghi, come attestato dall'esempio riportato di seguito: oggetto d'esame è questa volta il sermone recitato il 13 febbraio dedicato a "come dei avere nel cuore, nell'opere e nelle parole sempre il nome di Gesù, e come dei fugire le cagioni dé peccatì. Il sermone si presenta, ad una prima lettura, ricco di interrogative, giustificabili, forse, con la necessità di convincere i fedeli ad operare il bene, avendo sempre a mente l'esempio del Cristo; tale scopo spiega quindi il ricorso a una sintassi fortemente espressiva caratterizzata da continui cambiamenti nel tono di voce del predicatore. In questo contesto, Bernardino ricorre ad un'allegoria:

Se sarà uomo che abbia, alcuna volta, di quelle rabbie che vengono alcuna volta a' buoi, che si chiama l'assillo dice Iddio che quella persona di cui sono quegli si fatti buoi, si facci loro il protesto, e se infra due o tre di non lo legherà o ucciderà, che d'ogni danno facesse poi, sia tenuto di ristorare; e, uccidendo alcuna persona il bue, con colui di cui è, fusse morto. ${ }^{40}$

Segue quindi la spiegazione dell'allegoria, introdotta da una proposizione interrogativa cui se ne aggiunge, poi, un'altra, funzionale a precisare ulteriormente il significato dell'artificio retorico impiegato dal predicatore:

Chi è il bue? È il corpo nostro carnale con due corna, cioè con l'irascibile e concupiscibile. Chi è il Signore? È la ragione. Se col tuo irascibile o concupiscibile offendi alcuna persona, contro all'ordine del Signore, fatto il protesto due o tre volte, cioè che la ragione te lo contraddica, vuole la legge divina sia morto l'uno e l'altro, cioè l'avarizia disordinata e l'iracundia disordinata, e simile degli altri peccati. ${ }^{41}$ 
Come si vede, alle due interrogative, accompagnate dalle relative risposte, segue la spiegazione dell'intera allegoria che ne chiarisce una volta per tutte il senso esplicitando il diretto riferimento alla vita dell'uomo e al suo agire quotidiano.

In un altro sermone, recitato il 24 febbraio 1425 , lo stesso procedimento ricorre applicato a una metafora presente all'interno del vangelo di S. Marco:

Dice santo Marco che gli era già venuta la sera, e la navicella era nel mezzo del mare, e Gesù solo in terra, e vedeva che s'affaticavano in remicando, e'l vento era loro contrario. ${ }^{42}$

Seguono le interrogative con cui Bernardino si accinge a fornire una spiegazione del passo riportato: «Che significa il mare? Il pericolo. La nave? Uno giovane fragile co' buoni disiderii, in pericolo co' venti contrarii del dimonio». ${ }^{43}$

Il ciclo senese presenta un esempio molto interessante in cui si accumulano diverse proposizioni interrogative, funzionali a spiegare il senso di un'allegoria presente all'interno di una citazione scritturale. Il sermone in questione è quello recitato venerdì 22 agosto e dedicato all'esposizione dei peccati della lingua; la predica si apre con una citazione tratta dal salmo di Davide ( $D$ raco iste quem formasti ad illudendum ei: omnia ad te expectant») che aveva già costituito il thema dei sermoni tenuti nelle due giornate precedenti. All'esposizione del thema, segue immediatamente la traduzione in volgare: «Questo draco, questa maladetta bestia dice David-la quale tu, o Idio, hai formata perchénoice ne facciamo beffe; non di meno noi pure aspettiamo la grazia da te. A le grazie stamane». ${ }^{44}$ Dopo aver brevemente ricordato quanto già esposto nelle due prediche precedenti, Bernardino inserisce una citazione tratta da Daniele (14, 25-26): "Rex, da michi potestatem et interficiam draconem sine baculo et ense. Et dixit rex: do. Qui congregavit montem picis et adipis et capillorum etc.». Segue quindi la traduzione in volgare ("Dice Daniello: "O re, dammi potestà, e io ammazzarò questo dracone"») accompagnata dalla spiegazione dello stratagemma con cui Daniele riuscì ad uccidere il drago:

Elli ragunò uno monte di pece e uno monticello di sevo e un poca di borra, cioè dimolti peli di molte ragioni, dove potremo dire che ebbe dé capelli delle code delle donne; ebbe code d'asini e d'ogni ragione peli che elli poté avere, e mescolò insieme tutte queste cose, e di questa brodata fece una grande brigata di pallottole, le quali come l'ebbe fatte elli si fece incontra al dracone, e piglia queste pallottole, e a una a una tutte le gittò in bocca al dracone. Piglia l'una, piglia l'altra, tanto che lil dè tutte mangiare, e tanta fu la quantità delle pallottole, che s'empi sì il corpo, che elli crepò. ${ }^{45}$ 
A questo punto, al fine di decodificare il significato della complessa allegoria, Bernardino ricorre a una serie di interrogative volte a spiegare il senso del passo descritto:

A proposito, sai chi è il dracone velenoso? È il detrattore che sempre avelena col fiato delle sue parole. Chi è Daniello? È l'anima buona, la quale è detratta dal detrattore. E che fa questa anima buona, che fae? Ella piglia pece nera e sevo bianco, l'arme del Comuno di Siena, e piglia peli, e mescola queste cose insieme e fanne la brodata, e poi ne fa le pallottole. Che significa la pece? L'umiltà. E'l sevo? Carità del prossimo. E' peli? El conoscimento e lo intelletto di se medesimo e di Dio. ${ }^{46}$

Ogni singolo elemento presente nella descrizione del modo in cui Daniele uccide il drago è sottoposto a un processo di interpretazione che ne svela il significato ulteriore nascosto dietro quello letterale. Tutto il meccanismo di rivelazione del senso allegorico è basato sul ricorso alle proposizioni interrogative che costringono ad uno sforzo ermeneutico tanto il predicatore quanto i suoi ascoltatori.

\section{Proposizioni interrogative Retoriche}

Una delle classi più numerose è costituita dalle cosiddette domande retoriche, costruite con il preciso intento di predisporre la reazione degli ascoltatori e di orientarne, quindi, l'evoluzione del pensiero per giungere alla condivisione della tesi sostenuta dal predicatore. Interrogative di questo tipo ricorrono in tutti i cicli analizzati e costituiscono una costante dell'arte omiletica bernardiniana. Vediamone qualche esempio.

Nel sermone recitato il 10 marzo 1424, Bernardino è impegnato nell'esporre ai suoi ascoltatori i fattori che rendono nulla una confessione: "Del quale lume pure sopra la confessione tre sprendori si dimostrano. Il primo: iscusativa. Secondo: diminutiva. Terzo: purgativa» ${ }^{47}$. Relativamente alla seconda causa, Bernardino afferma che può capitare che il fedele debba confessarsi nuovamente di un peccato già confessato; ciò avviene ad esempio quando il confessore non è in grado di valutare con esattezza i peccati commessi e li giudica in maniera sommaria:

Piglia per regola generale che se 'l confessoro non sa discernere e tuoi peccati e passagli cosi alla grossa, non se' confessato. Se chi t'avessi a tegnere un pezzo di panno e non sapesse conducerlo e guastasselo, non è egli tenuto a rifartelo?Maisi.48 
Per dimostrare l'evidenza della sua affermazione, Bernardino ricorre a un esempio concreto e lo propone ai suoi ascoltatori sotto forma di interrogativa retorica in grado di avvalorare la sua tesi dimostrandone la veridicità.

Un procedimento simile si ritrova anche nella predica recitata il 21 marzo 1424, dedicata al tema del sacrilegio; nel sostenere l'obbligo di reverenza nei confronti del clero, anche quando i suoi rappresentanti commettono peccati, Bernardino ricorre ad un paragone presentato tramite una domanda retorica:

Sono statuiti come sacerdoti, capo degli altri, e come el nostro capo, è sopra a tutte le nostre membra, non si ispicchi mai la riverenza. [...] Se si cacciassino tutti e cattivi, pochi rimarebbono e buoni, egli è meno male avergli non buoni che non avere niuno. Se tu avessi guasta una mano, mozzerestiti tu el capo per guarire della mano? Mainò. Ese avessi rotto el capo mozzerestiloti tu per non averlo rotto? Mainò! Egli è meglio capo rotto che capo tagliato. E preti cattivi sono i capi rotti, e loro è il danno ${ }^{49}$.

È possibile individuare casi simili nel secondo ciclo fiorentino dove, per esempio, nel sermone recitato il 7 marzo, Bernardino ricorre a una interrogativa retorica al fine di porre in rilievo la grandezza della ricompensa divina:

Se tu fai uno servigio a uno tuo parente o amico, ed egli te n'usa gratitudine, nol servi tu poi un'altra volta più volentieri? Mai sì! Se questo interviene tra noi mortali, non credere che Iddio, quando vedrà tu sia grato de' suoi benefici, non ti facci di nuovo maggiormente bene, massime perch'egli è creatore degli uomini ${ }^{50}$.

Ancora, nel sermone dedicato alla repressione del vizio della lussuria si legge: "Non è ben pazzo colui che mette fuoco nella sua casa medesima? Se antiporrai la divozione di messer Domenedio a quella della carne, andrà bene e sarai franco" ${ }^{51}$.

Il ciclo senese, con il suo ricco patrimonio espressivo, non fa eccezione ed è possibile individuare diversi esempi di proposizioni interrogative retoriche. Si prenda la predica tenuta il 4 settembre dedicata al tema "dell'ordinato amore che debba essare infra la moglie e 'l marito». Sottolineando l'importanza del ruolo svolto dal predicatore nell'evitare che gli uomini incorrano nel peccato o nel consigliare loro la via per uscirne, una volta commessi, Bernardino si rivolge al suo pubblico e chiede: 
Doh! Vuoi tu vedere sio so' tenuto amunirvi del peccato vostro? Or considera: non so' io tenuto cogli altri religiosi di trarre l'anime vostre delle branche del demonio, giusto a mio potere.52

Per avvalorare maggiormente la sua posizione, Bernardino istituisce un paragone fra la sua azione in qualità di predicatore e la sua azione in qualità di cittadino, dotato di senso civico e amore non solo nei confronti della sua città ma anche e soprattutto nei confronti del suo popolo:

Ma ditemi: se io vedesse colà uno che volesse avelenare quella fonte, se io potesse stroppiare che elli non l'avelenasse colle mie parole, nol debbo io stroppiare? E se pure ella fusse stata avelenata, non so' io tenuto a dirvelo, che voi non ne beiate, accio che voi non siate avelenati? Certo si ${ }^{53}$.

Un altro esempio si trova nel sermone recitato il 15 settembre dove Bernardino intende sottolineare il valore del rapporto personale che il fedele deve istituire con la Vergine Maria:

Sappi che come tu saluti la Vergine Maria, subito ella saluta te. Non pensare che ella sia di queste rusticacce come assai se ne truovano: anco è tutta piacevole. Ella ti saluta ogni sera quando tu senti sonare l'avemaria quassù in Vescovado. O sarai tu tanto villano, che tu non saluti lei? Come tu la salutarai, subito ella ti rispondará ${ }^{4}$.

Poco dopo, con procedimento simile, Bernardino mette in luce l'onestà della Vergine:

La siconda damigella che era con Maria, si si chiamava madonna Onesta. [...] Credi tu forse che ella s'adornasse come voi altre fate? No. Come Iddio l'aveva fatta, cosi si stava. ${ }^{55}$

\section{Proposizioni InTERrogative CON FUnZIONE FÀtica}

Una delle funzioni identificate da Roman Jakobson ${ }^{56}$ come caratteristiche di un discorso è quella che va sotto il nome di funzione fatica. Si tratta di un tipo di funzione che ricorre ogni volta che si rivolge particolare attenzione al mezzo attraverso il quale il messaggio viene veicolato. Per tale ragione si esplica spesso in proposizioni prive di un vero e proprio contenuto semantico e funzionali

52 S27, XX, vol. I, p. 575.

53 S27, XX, vol. I, p. 575.

54 S27, XXX, vol. II, p. 866.

55 S27, XXX, vol. II, p. 875.

56 R. Jakobson, Saggi di linguistica generale, a cura di L. Heilmann, Feltrinelli, Milano, 1966 
ad accertare il persistere del contatto che mette in comunicazione mittente e destinatario del messaggio. Si prenda ad esempio il caso di una conferenza nel corso della quale il locutore principale si rivolge al proprio uditorio chiedendo: «Mi sentite?». Nel formulare tale domanda, egli sta esercitando un controllo sul mezzo di comunicazione che gli permette di divulgare il proprio discorso; in altre parole sta verificando l'esistenza del contatto, responsabile della trasmissione del messaggio.

Nel caso dei sermoni bernardiniani è possibile attribuire una funzione fatica ad alcune proposizioni interrogative che ricorrono con una frequenza tale da «configurarsi come un intercalare, e quindi di assolvere, in certo qual modo, alla precisa funzione di stabilire e soprattutto mantenere la comunicazione» ${ }^{57}$. Espressioni quali, ad esempio, "Sammi intendere?" 58 "Hami inteso?» 59 , "Avetemi voi inteso, donne?» ${ }^{60}$ testimoniano il continuo accertarsi dell' esistenza del contatto.

\section{Proposizioni interrogative Correttive}

L'ultima delle classi oggetto di analisi racchiude una serie di domande che Bernardino sembra rivolgere a sé stesso esplicitando i dubbi sorti nella sua mente a seguito delle parole pronunciate. Si tratta di domande definite da Piero Sollazzi «correttive» in quanto, molto spesso, mettono in luce la rettifica di quanto il predicatore va affermando, a beneficio di una riformulazione del suo pensiero. Tali interrogative ricorrono, per la maggior parte, nel ciclo senese anche se non è raro trovarne qualcuna anche nei cicli fiorentini precedenti. Di seguito alcuni esempi:

Tu sai che molte volte è stato detto, insino quando ero fanciullo udii che Antecristo era nato. Ma che dico io? Insino al tempo delli apostoli si disse ch'elli era nato, e anco al tempo di santo Bernardo. (S27, III, vol. I, p. 152)

Cosi talvolta la virtù della sapienzia delle cose del mondo. Unde Gregorio (VI, Quaestio prima): «Sunt plurimi de quibus tot virtutes et bona dicuntur, quod non est in eis undecima pars. Dico che so' molti, molti dico... (Che ho io detto? Che ho detto?)... So' molti che dicono tanto bene d'uno, che non è delle mille parti l'una di quello che si dice» (S27, VIII, vol. I, pp. 290 - 291)

L'ultimo esempio è particolarmente interessante in quanto, leggendo il sermone, si ha come l'impressione che il predicatore abbia perso il filo del suo discorso; la domanda sembra quindi rilevare la presa di coscienza dello smarrimento da parte di Bernardino che, dopo un attimo di esitazione, sembra tornare padrone della situazione recuperando il senso logico del suo sermone.

57 P. Sollazzi, Meccanismi allocutivi in San Bernardino...cit., p. 424.

58 F25, XLI, vol. II, p. 367.

59 S27, XVI, vol. I, p. 466.

60 S27, XIX, vol. I, p. 560. 
LE PROPOSIZIONI INTERROGATIVE: ANALISI LINGUISTICO - SINTATTICA

Esaurita l'esposizione della classificazione per categorie, utile a mettere in rilievo le funzioni svolte, dalle proposizioni interrogative, si rende necessaria una breve analisi linguistico - sintattica che ne evidenzi le principali caratteristiche formali.

\section{PRONOMI}

La maggior parte delle proposizioni interrogative sono rivolte a un interlocutore identificato dal pronome di seconda persona singolare, esplicito o implicito:

- Epogniamo che tu sia confessato per la pascua di resurresso bene e diligentemente e abbi preso il corpo di Gesù Cristo, e valicato otto o quindici di o un mese e tu caggi in veruno peccato mortale e pentitene, debbi tu indugiare in capo dell'anno, cioè a l'altra quaresima che viene a confessarti? (F24, III, vol. I, p. 34)

- Se tu vuoi distinguere e separare l'uso dal fiorino come farai? (F25, XLI, vol. II, p. 369)

- Vuoi tu chio tinsegni a conoscere chi è atta a far bene e ha qualche poco di sentimento? (S27, XXX, vol. II, p. 879)

- Se non la udirai come la saprai? (F24, XLI, vol. II, p. 219)

- Perché credi si vergogni più dell'atto della lussuria che di niun altro peccato per grande peccato che sia? (F25, XLVII, vol. III, p. 44)

- Sai il proverbio? (S27, IX, vol. I, p. 307)

Con frequenza minore ricorrono anche proposizioni dirette a interlocutori plurali in cui, analogamente a quanto visto nel caso precedente, il pronome di seconda persona può essere esplicito o implicito:

- A che fare portate voi tutte le cose e le fogge che portano le meritrici? (F24, XXXVII, vol. II, p. 141)

- Quanti peccati fate voi di quegli che voi non faresti, se credessi fusse peccato? (F25, XVIII, vol. I, p. 325)

- Sapete perché? (S27, XXX, vol. II, p. 875)

- Doh, ditemi: non sete voi cristiane? (S27, XXXVII, vol. II, p. 1071)

Non mancano le attestazioni di interrogative alla terza persona, per lo più al singolare, del tipo:

- Ecci niuna che vi sia cascata, ecci niuna ruffiana? (S27, XVI, vol. I, p. 471)

- Ecci niuna qui fra voi che sia contenta, o niuno che sia contento? ( S27, VIII, vol. I, p. 286)

- Ecci niuna fornaia? Or attende [...] ( S27, IX, vol. I, p. 315) 
In alcuni casi è possibile registrare un cambio nel pronome di riferimento con passaggi, per esempio, dalla seconda persona plurale alla seconda persona singolare: "O donne, vedeste voi mai impiombare le paglie? Sai come simpiombano, e chi è colui che le 'mpiomba? ${ }^{61}$

A volte, infine, il predicatore rivolge la domanda a un "noi" collettivo che lo coinvolge anche in qualità di semplice cittadino:

- Per che cagione non vuole che noi el sappiamo? ( S27, IV, vol. I, p. 200)

- Che frutto avemo di questa resurressione? (F25, LXIV, vol. III, p. 410)

\section{FORMULE INTRODUTTIVE}

Le diverse proposizioni interrogative esaminate finora sono generalmente introdotte da una serie di procedimenti linguistici particolarmente espressivi, capaci di segnalare la presenza di un dialogo. I «demarcativi di apertura dialogica», ${ }^{62}$ descritti da Carlo Delcorno nella sua analisi della sintassi di Giordano da Pisa, ricorrono con analoga funzione anche all'interno del corpus bernardiniano.

In primo luogo è possibile individuare una formula verbale costituita dall'unione della seconda persona del verbo 'sapere' con diversi avverbi o pronomi interrogativi, combinati in forme del tipo: «sai che...» «sai come...?», «sai perché...?», «sai quando...?», «sai qual è...?»" ${ }^{63}$ Vediamone qualche esempio:

- Esai come è scritto nel Vangelio? (F24, II, vol. I, p. 17)

- Sai quale è il giuramento doloso? (F25, XXIX, vol. II, p. 153)

- Sai perché la pose più nella faccia che in altro luogo? (S27, IX, vol. I, p. 316)

- E sai che seguita poi di queste cotali buone? (S27, XXII, vol. I, p. 645)

- Sai quando non ti senti divozione di confessione? (F25, XXXVII, vol. II, p. 303)

Altrettanto frequente è la formula introdotta dal verbo 'volere' coniugato alla seconda persona, singolare o plurale:

- Vuoi tu fare bene a te? Mai si!' (F24, XLII, vol. II, p. 236)

- Volete voi sapere se voi dovete capitare male e rovinare nellira di Dio? (F24, XXVI, vol. I, p. 433)

- Vuo' tu avere vittoria? (F25, IX, vol. I, p. 167)

- Vuoi vedere come la cosa andarà? (S27, XXXVII, vol. II, p. 1087)

61 S27, VII, vol. I, p. 270

62 C. Delcorno, Il "parlato" dei predicatori. Osservazioni sulla sintassi di Giordano da Pisa, in «Lettere Italiane», LII, (2000), pp. 3 - 50, p.43.

63 Per l'analisi formale delle proposizioni interrogative si rinvia al testo di P. Sollazzi, Meccanismi allocutivi in S. Bernardino...cit., p. 422, utilizzato come base dell'esame proposto. 
- Volete voi altro? (S27, XXXVII, vol. II, p. 1081)

- Ma vuoi tu vedere quando è contrizione? Io te lo insegnerò (F24, X, vol. I, p. 153)

Molto significativo è anche il ricorso alle formule interrogative introdotte dal verbo 'vedere' cui segue, nella maggior parte dei casi, il pronome esplicito di seconda persona singolare:

- Non vedi tu che l'acqua piglia la forma del vaso ov'ella è messa, e questa si è per esperienza la gioventudine che più tosto si contaminano che gli altri? (F24, XXXI, vol. II, p. 43)

- Non vedi tu che quando l'uomo pecca mortalmente, era prima innocente $e$, fatto il peccato, perde quella innocenza e, se torna a penitenza, mai più racquista quella innocenza? (F25, XXV, vol. II, p. 84)

- Vedi tu come fa lo scarafaggio? (S27, VII, vol. I, p. 268)

In alcuni casi, l'invito a 'vedere' è funzionale all'attualizzazione del discorso evangelico tramite il riferimento a elementi concreti, propri della vita quotidiana dei fedeli (es. riferimento all'acqua o allo scarafaggio). Si tratta di uno stratagemma che consente al predicatore di realizzare quella riduzione a sermo humilis, principio cardine della teoria omiletica bernardiniana.

Non è raro, inoltre, trovare formule introdotte dal verbo 'credere', di solito in combinazione con il pronome 'quanto':

- Quanto credi che se ne dolesse, e massimamente a considerare le pene sanza fine? (F24, XLV, vol. II, p. 286)

- Quanto credi ch'ella meritasse nella vita sua? (S27, XXIV, vol. I, p. 688)

- O li apostoli che so' ora beati, quanto credi che lo intendino piu ora che quando erano in carne mortali? (S27, XXIV, vol. I, p. 690)

- Quanto credi che egli lo intendesse meglio che noi? (S27, XXIV, vol. I, p. 697)

Ovviamente, come si è già avuto modo di notare, molte interrogative sono introdotte dal verbo 'dire' usato alla seconda persona singolare o plurale del modo imperativo: "dimmi/ditemi». Spesso, tale verbo è preceduto da un'interiezione tipica di Bernardino, «Doh», usata anche in contesti diversi da quelli puramente interrogativi:

- Doh! Dimmi: non sai tu la regola e 'l modo che tu hai a tenere? (S27, VII, vol. I, p. 266)

- Doh, dimmi: quanti testamenti so' stati fatti da coloro che so' ora passati, e hanno lassato che si facci tale e tal cosa, e tu esci fuore de la volontà del testatore? (S27, XXXV, vol. II, p. 1028) 
- Doh, dimmi: hai tu veduta Italia come ella sta nel Lappamondo? (S27, XXXIX, vol. II, p. 1145)

Il riferimento a questa interiezione offre lo spunto per segnalare la presenza di alcuni "demarcativi di apertura dialogica" che svolgono una "funzione di segnale» ${ }^{64}$ del dialogo che sta per prendere avvio. Si tratta di avverbi e interiezioni che preannunciano la formulazione di una domanda e, dunque, di una costruzione di tipo dialogico. Fra quelli più frequenti possiamo segnalare:

1) l'avverbio Or, ora:

- Or che ci mancherà elli ecc? (F24, XI, vol. I, p. 70)

- Or che ti giova la fatica dell'allevallo, del nutricarlo, se sé cagione di mandarlo allo inferno? (F25, XLVII, vol. III, p. 42)

- Or non vedete voi che ogni uomo e ogni donna, ogni fanciullo, insino alle frutta, avete fatto che sieno guelfi o ghibellini? (S27, X, vol. I, p. 331)

2) le congiunzioni $e, o$ :

- E quando è che non può assolverti? Quando tu ti confessi a un altro prete che non sia prete del tuo popolo [...] (F24, III, vol. I, p. 36)

- E che credi che fusse questa trasfigurazione? (F25, XXIX, vol. II, p. 165)

- $O$ che vale crescere di bene in meglio se tu non perseveri insino alla fine? Niente! (F25, XLIX, vol. III, p. 84)

- E sai di che io ti voglio dire? (S27, IV, vol. I, p. 176)

- O che fa il gattivo? (S27, VII, vol. I, p. 257)

3) il dunque iniziale:

- Dunque di che aviamo noi a parlare stamane? (S27, XVI, vol. I, p. 462)

- Dunque che voliamo tanto parlarne? (S27, XXIV, vol. I, p. 697)

- Dunque se elli el meritava come Lucifero, perché non fu dannato? (S27, XXIV, vol. I., p. 704)

4) l'interiezione iniziale doh:

- Doh, che direte di quello chio vi dirò? ( S27, IV, vol. I, p. 187)

- Doh, voliamo noi vedere quando Idio manda i suoi giudici? (S27, XIII, vol. I, p. 389)

- Doh, non considerate voi a quello che Idio ha fatto? (S27, XX, vol. I, p. 591)

\section{Conclusioni}

L'esame delle interrogative, quali si configurano all'interno del corpus omiletico bernardiniano, ha condotto all'isolamento di diverse classi di proposizioni,

64 C. Delcorno, Il parlato.....cit., p. 43. 
distinte in base a specifiche funzioni e caratteristiche. Al di là delle peculiarità proprie di ciascun raggruppamento, è possibile svolgere alcune considerazioni relativamente al ruolo svolto dalle interrogative ai fini della strategia comunicativa bernardiniana. In primo luogo, essendo dotate di una grande forza espressiva, tali proposizioni contribuiscono a rendere il sermone più attraente, evitando la disattenzione e la noia degli ascoltatori. Secondariamente, in qualità di atti illocutori, interrompono il monologo del predicatore introducendo nel discorso una struttura dialogica capace di stimolare la partecipazione del pubblico; in questo modo i fedeli da semplici ascoltatori passivi vengono investiti di un ruolo e di una funzione che li rende attivi e partecipi alla comunicazione loro rivolta. Alla luce di tale dato si comprende perché l'analisi della presenza, della frequenza e della tipologia di proposizioni interrogative utilizzate in un testo concepito per la diffusione orale costituisce un elemento utile a determinare il grado di teatralità implicita del sermone. Interrompendo il discorso del predicatore e introducendo il riferimento a un altro interlocutore, le interrogative trasformano la comunicazione unidirezionale del predicatore in dialogo, elemento tipico del teatro.

D'altra parte, il ricorso alle proposizioni interrogative è del tutto coerente con il programma evangelico bernardiniano: il desiderio di offrire ai suoi fedeli modelli di comportamento applicabili alla vita quotidiana e insegnamenti utili a non incorrere nel peccato spinge, infatti, il santo a servirsi di uno stile capace di istituire un dialogo costante con i suoi ascoltatori. Per tale ragione Bernardino è attento a confezionare un messaggio in cui brani altamente dottrinali si alternano a brani più colloquiali e capaci, per questo, di catturare l'attenzione del pubblico. E proprio la necessità di mantenere attenti i suoi ascoltatori, spiega il continuo impiego di proposizioni interrogative in grado di riconfermare costantemente il rapporto fra predicatore e fedeli affinché la predica da monologo si trasformi, anche solo apparentemente, in dialogo. 
